\title{
Reviews
}

\section{Angiogenic markers in pre-eclampsia}

\author{
Walfrido W Sumpaico ${ }^{1}$ \\ Sri Lanka Journal of Obstetrics and Gynaecology 2009; 31: 48-52
}

Key words: Angiogenic markers, pre-eclampsia.

\section{Introduction}

Every year, 536,000 women die from pregnancyrelated causes. More than 80 percent of maternal deaths worldwide are due to five direct causes: hemorrhage, sepsis, unsafe abortion, obstructed labor and hypertensive disease of pregnancy ${ }^{1}$. The latest report from the Confidential Enquiries into Maternal Deaths (UK) lists eclampsia and pre-eclampsia as the second most common cause and showed an increase from the previous report ${ }^{2}$. The latest statistics from the Philippine Obstetrical and Gynecological Society (2006) listed hypertension as causing 143/545 (26.24\%) maternal deaths. Further broken down, hypertension deaths were preeclampsia (50), eclampsia (66), pre-existing hypertension (8), chronic hypertension with pre-eclampsia (8) and HELLP syndrome $(11)^{3}$. Clearly, therefore, any attempt to curb maternal and perinatal mortality and morbidity due to hypertensive states will result not only in less loss of lives but also in less deprivation of financial and material resources. This paper presents an update on a new pseudo-vasculogenesis theory and its possible application as diagnostic markers in the prediction and prevention of pre-eclampsia.

\section{Pathophysiology of pre-eclampsia}

Numerous theories have been advanced regarding the cause(s) of PIH and the reader is referred to the book of Chesley ${ }^{4}$ for a historical review of these.

"...Everyone from allergists to zoologists has proposed hypotheses and suggested radical therapies based upon them such as mastectomy, oophorectomy, renal decapsulation, trephination, alignment of the patient with the earth's magnetic field with her head pointing toward the North Pole and all sorts of medical regimens."

(Leon Chesley, 1971)

Current data indicate that placental ischemia and trophoblastic hypoperfusion with endothelial

\footnotetext{
${ }^{1}$ Professor, Department of Obstetrics and Gynecology, MCU-FDT Medical Foundation, Caloocan City, Philippines.

E-mail:wwwsumpaico.aofog.org
}

dysfunction is the most consistent change in preeclampsia and is believed to be the pivotal insult in this disease.

\section{Pseudo-vasculogenesis Theory}

A pathophysiological study ${ }^{5}$ has examined the hypothesis that placental ischemia occurs at an early stage and upregulates placental production of a soluble anti=angiogenesis protein called soluble fmslike tyrosine kinase 1 (sFlt1) that leads to maternal endothelial dysfunction and its clinical sequelae of hypertension, proteinuria, and edema. SFlt1 acts as a potent antagonist to vascular endothelial growth factor (VEGF) and placental growth factor (PlGF) which help to reduce vascular tone and blood pressure (pro-angiogenesis).

The study further showed the following findings:

1. total serum sFlt1 was nearly $5 x$ higher in those with severe preeclampsia than in normotensive women, and this difference was not explained by earlier gestational age.

2. serum ELISA levels of free VEGF and PIGF were significantly reduced in the presence of recombinant sFlt1.

3. Angiogenesis, as reflected by endothelial tube formation, was inhibited by serum from women with preeclampsia, and an analogous effect was noted in vitro when sFlt1 to was added serum from normotensive women. Adding VEGF and PlGF to preeclamptic serum restored endothelial tube formation. An in vitro assay for microvascular reactivity showed that sFlt1 blocked the dosedependent increase in vasodilation produced by VEGF or PIGF.

4. Most importantly, recombinant adenovirus encoding the murine sFlt1 gene product produced significant hypertension and heavy albuminuria when injected into pregnant rats. Histopath examination in the pregnant rats treated with sFlt1 showed renal glomeruli were enlarged and capillary loops were occluded by swollen, hypertrophied endocapillary cells. These findings suggest a cause and effect relationship that excessive placental production of sFlt1 contributes 
to hypertension, proteinuria, and glomerular endotheliosis in preeclamptic women.

Another protein factor in pseudo-vasculogenesis is soluble endoglin. It is upregulated in syncytiotrophoblasts and its levels are exaggerated during and, significantly, prior to the onset of preeclamptic symptoms. Levine et al have recently demonstrated a 2-3x endoglin increase in preterm and term pregnancy, respectively, compared to non-pregnant states. Significantly, mild preeclampsia, severe preeclampsia, and HELLP syndrome are accompanied by a further three-, five-, and ten-fold increase in circulating endoglin, respectively. These elevated endoglin levels closely parallel increases in sFlt-16.

What causes the upregulation of sFlt1 and other anti-angiogenesis proteins? Fisher, et al 7 offers an explanation that placental hypoxia (decreased oxygen) serves as the driving force for the phenomenon of "pseudovasculogenesis" (Figure 1). This process can be described as follows:

1. The early stages of placental development take place in a relatively hypoxic environment that favors cytotrophoblast proliferation rather than differentiation along the invasive pathway. Accordingly, this cell population (green cells) rapidly increases in number as compared with the embryonic lineages.

2. As development continues, cytotrophoblasts (dark green cells) invade the uterine wall and plug the maternal vessels, a process that helps maintain a state of physiological hypoxia. As indicated by the blunt arrows, cytotrophoblasts migrate farther up arteries than veins.

3. By 10 to 12 weeks of human pregnancy, blood flow to the intervillous space begins. As the endovascular component of cytotrophoblast invasion progresses, the cells migrate along the lumina of spiral arterioles, replacing the maternal endothelial lining. Cytotrophoblasts are also found in the smooth muscle walls of these vessels. In normal pregnancy the process whereby placental cells remodel uterine arterioles involves the decidual and inner third of the myometrial portions of these vessels. As a result, the diameter of the arterioles expands to accommodate the dramatic increase in blood flow that is needed to support rapid fetal growth later in pregnancy. It is likely that failed endovascular invasion leads, in some cases, to abortion, whereas an inability to invade to the appropriate depth is associated with preeclampsia and a subset of pregnancies in which the growth of the fetus is restricted.

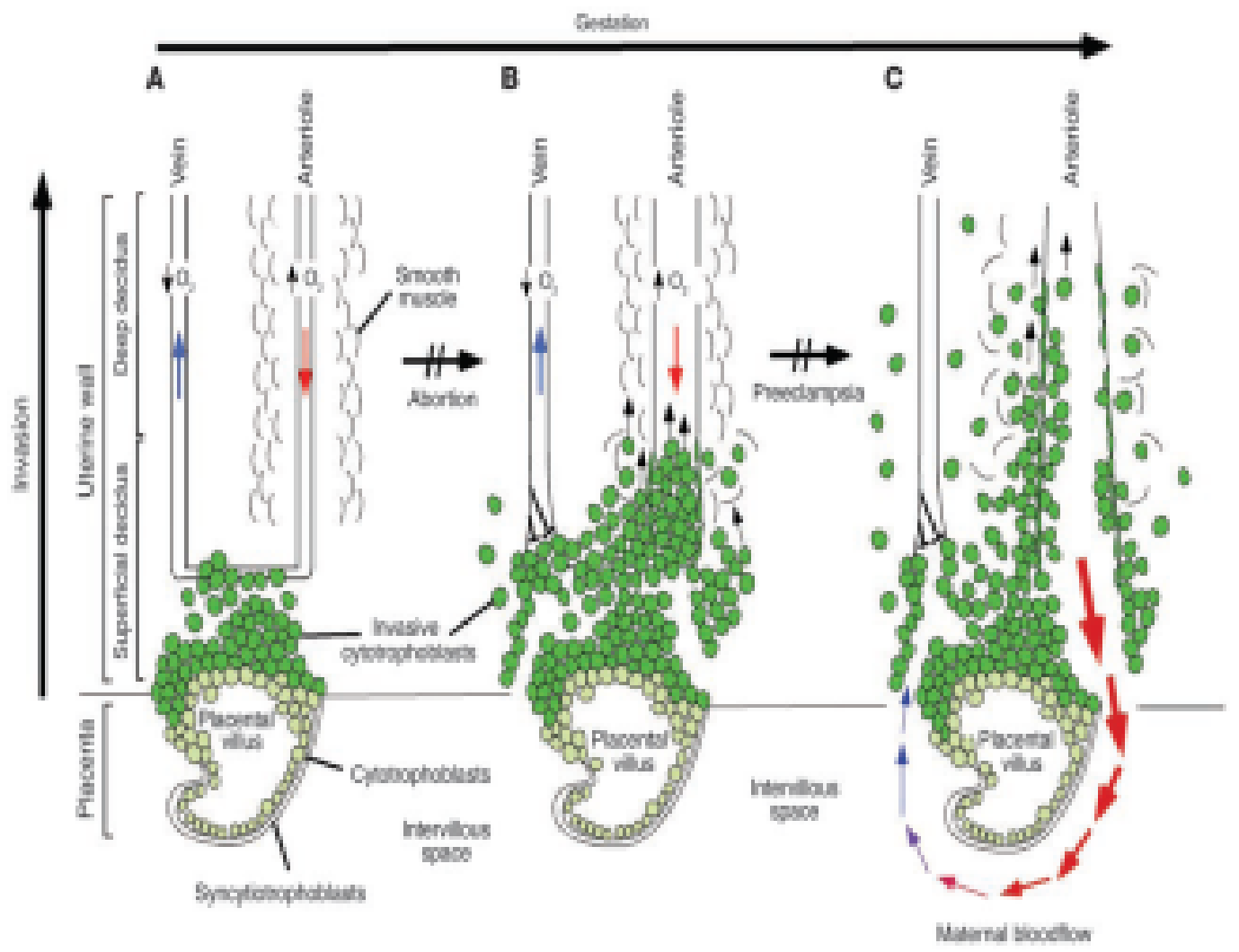

Figure 1. Pseudovasculogenesis and placental hypoxia (Fisher et $\mathrm{al}^{7}$ ) 
A summary diagram shows the current thinking that pre-eclampsia may be considered a 2-stage disease (Figure 2).

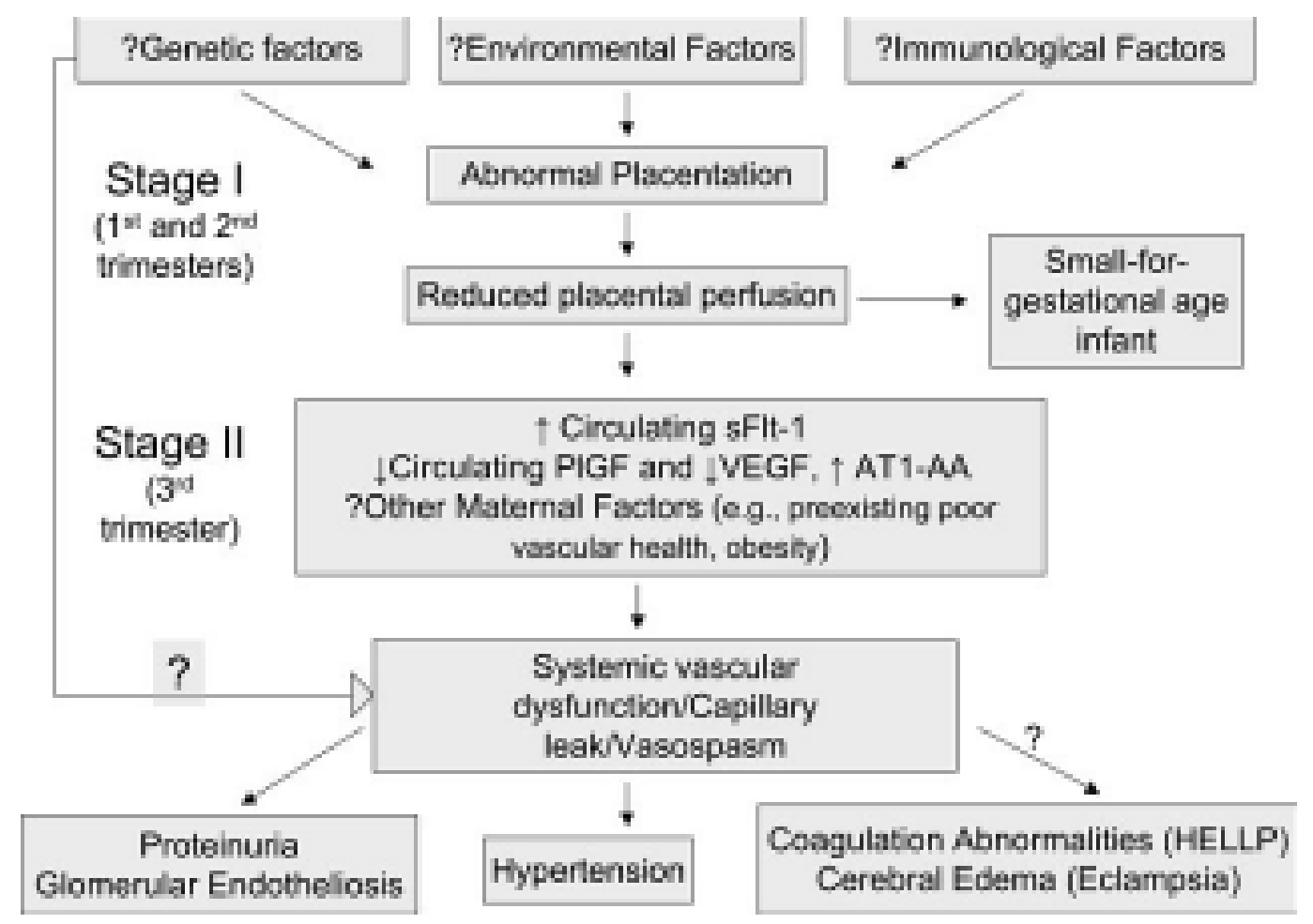

Figure 2. Pre-eclampsia a 2-stage disease

\section{Clinical studies on angiogenic markers}

sFlt-1. To evaluate gestational patterns, Levine et al performed a cross-sectional analysis of serum obtained within gestational-age intervals of four to five weeks (Figure 3A). The sFlt-1 concentrations in controls remained constant until 33 to 36 weeks of gestation, when they increased by approximately 145 pg per milliliter per week until labor and delivery. In the women in whom preeclampsia later developed, but before the onset of preeclampsia, the concentrations began to increase at 21 to 24 weeks of gestation, with a steeper increase at 29 to 32 weeks. When they compared the sFlt-1 concentrations at different stages of pregnancy in specimens from women in whom preeclampsia developed, they found that among women with specimens drawn when their fetuses were in the same gestational-age interval, those who already had clinical preeclampsia had significantly higher concentrations than those who did not (Figure 3A).

The concentrations of sFlt-1 were increased in women who later had preeclampsia beginning 11 to 9 weeks before the onset of preeclampsia. Five weeks before the onset of preeclampsia, the average concentration in women in whom preeclampsia developed was similar to the concentrations in controls at term, but after this point the sFlt-1 concentration in the women with preeclampsia was higher (Figure 3B).

\section{Placental growth factor}

The PlGF concentrations in the controls increased during the first two trimesters, peaked at 29 to 32 weeks, and decreased thereafter. The PlGF concentrations in the women with preeclampsia followed a similar pattern but were significantly lower than those in the controls from 13 to 16 weeks onward (Figure 4A).

Similar to s-Flt- 1 , the PlGF concentrations began to decrease 11 to 9 weeks before the onset of preeclampsia, with substantial reductions during the 5 weeks before the onset of hypertension or proteinuria (Figure 4B).

The key points of these landmark articles are:

- The pro-angiogenetic markers, PIGF and VEGF, both decrease with pre-eclampsia compared to normotensive women. 
Figure 4B. Mean PIGF concentration and weeks before preeclampsia

- The anti-angiogenetic maker, sFlt-1, increases with preeclampsia compared to normotensive controls.

- The decrease in PIGF and the increase in s-Flt-1 are evident 5 weeks before the onset of clinical pre-eclampsia.

There have been numerous articles which have validated these initial findings on angiogenetic markers and will not be mentioned here.

\section{Future directions}

Some articles have also done studies on other markers (soluble endoglin, PAPP-A, PP-13 or placental protein-13, podocyturia) and are worth remembering.

The use of ratios between the pro- and the antiangiogenetic markers have also been undertaken.

Studies using the combination of angiogenetic marker testing in the 1st trimester combined with uterine artery Doppler investigation (presence of a diastolic notch and pulsatility indices) are certainly worth looking into.

\section{References}

1. WHO/UNICEF/UNFPA maternal mortality estimates from 2000.

2. Lewis $G$ (Ed) 2007. The Confidential Enquiry into Maternal and Child Health CEMACH, Saving Mother's Lives: reviewing maternal deaths to make motherhood safer 2003-2005. The 7th Report on Confidential Enquiries into Maternal Deaths in the United Kingdom.

3. Philippine Obstetrical and Gynecological Society. Annual Reports of the Committee on Nationwide Statistics, 2006.

4. Chesley LC. Hypertensive Disorders in Pregnancy. New York, USA: Appleton-Century-Crofts, 1978.

5. Maynard SE, Min JY, Merchan J, Lim K, Li J, Mondal S, Libermann TA, Morgon JP, Sellke FW, Stillman IE, Epstein FH, Sukhatme VP, Karumanchi SA. J Clin Invest 2003; 111: $649-58$.

6. Levine RJ, et al. Circulating angiogenic factors and the risk of preeclampsia. N Engl J Med 2004; 350: 672-83.

7. Levine RJ, et al. Soluble endoglin and other circulating antiangiogenic factors in pre-eclampsia. N Engl J Med 2006; 355: 992.

8. Red-Horse K, Zhou Y, Genbacev O, Prakobphol A, Foulk R, McMaster M, Fischer SJ. Trophoblast differentiation during embryo implantation and formation of the maternal-fetal interface. JCI 2004; 114(6), 744-54. 